\title{
X-ray Variability of the Flare Star CC Eri
}

\author{
H. C. Pan, C. Jordan \\ Department of Physics (Theoretical Physics), University of Oxford, 1 Keble Road, \\ Oxford OX1 3NP, UK
}

The flare/spotted spectroscopic binary star CC Eri (HD 16157) was observed in the periods $1990 \mathrm{July} 9-11$ and 1992 January 26-27 with the ROSAT PSPC detector (Pan and Jordan 1995). These high quality data give the first information on the temporal and spectral variability of CC Eri in the X-ray energy band.

During the ROSAT observations the $\mathrm{X}$-ray intensity of the source is variable on timescales from a few minutes to several hours. The $\mathrm{X}$-ray luminosity is in the range $2.5-6.8 \times 10^{29} \mathrm{erg} \mathrm{s}^{-1}$, which is similar to values found from previous measurements with the Einstein IPC and EXOSAT LE. On 1990 July 10 an $\mathrm{X}$-ray flare-like event was detected with an exponential rise time of about one hour and a decay time of about two hours. The X-ray spectrum of CC Eri can be described by current thermal plasma codes with two temperature components or with a continuous temperature distribution. The spectral results show that plasma at $T_{\mathrm{e}} \sim 10^{7} \mathrm{~K}$ exists in the corona of CC Eri. We find that the X-ray spectrum is also variable and the variations of the emission measure, and to a lesser extent of the temperature, are correlated with the source intensity.

The X-ray variability of CC Eri may in principle be caused by flaring events and/or by the rotational modulation of active regions. However, rotational modulation of a spot related active region requires an unphysically large $\mathrm{X}$-ray flux in a concentrated area. Thus it seems more likely that some sort of flare was observed. The variation in the observed source flux can be reproduced by adopting a magnetic reconnection model. Comparisons with an unheated model, towards the end of the flare, suggest that the area and volume of the flare are substantially larger than in a solar two ribbon flare, while the electron pressure is similar. The larger flare area on CC Eri, compared with the Sun, may not be surprising given the larger optical starspot area. Thus a two ribbon flare provides one possible explanation of the variations observed. The emission measure and temperature of the non-flaring emission, interpreted as the average corona, lead to an electron pressure similar to that in a well-developed solar active region.

\section{References}

Pan H. C., Jordan C., 1995, MNRAS 272, 11 